\title{
Striations in a Radio Frequency Planar Inductively Coupled Plasma
}

\author{
J. A. Stittsworth, Student Member, IEEE, and A. E. Wendt, Member, IEEE
}

\begin{abstract}
Photographic evidence is presented for azimuthal structure in cylindrical radio frequency (RF) planar inductively coupled plasmas. Observations made in a high aspect ratio chamber (antenna radius large compared to height of plasma volume) show azimuthal striations (periodic modulations in emitted light intensity) in an argon discharge. These striations, reported here for the first time, have serious implications for discharge uniformity under some conditions (discharge shown at $300 \mathrm{~W}$ RF power, 85 mtorr). A comparison to positive column striations in de glow discharges shows some similarities, although a complete explanation will require consideration of RF effects.
\end{abstract}

\section{INTRODUCTION}

C YLINDRICAL planar radio frequency (RF) inductively coupled plasmas [1] (ICP) as used in commercial reactors for materials processing are generally considered both experimentally and theoretically as two-dimensional (2-D) $(r-z)$ systems, with symmetry in the azimuthal coordinate. In this paper, we offer photographic evidence that planar ICP discharges can exhibit dramatic azimuthal structure. Specifically, we observe azimuthal striations in the intensity of light emitted from an argon plasma glow, corresponding to azimuthal variation in the rate of neutral excitation due to electron impact. These striations may have significant implications for discharge uniformity and overall discharge behavior, particularly in large aspect ratio (radius/height) sources.

\section{DESCRIPTION OF EXPERIMENT}

The argon plasma observed in this study was confined to a cylindrical volume $36 \mathrm{~cm}$ in diameter by $2.5 \mathrm{~cm}$ high, inside a much larger vacuum chamber. The volume is bounded at one end by a quartz vacuum window and at the other by an aluminum plate with a $1-\mathrm{cm}$ wide radial slot for diagnostic access. The radial boundary is defined by a cylindrical tube made of perforated aluminum sheet. The antenna used for inductive power coupling to the plasma is a $28-\mathrm{cm}$ diameter single-turn circular loop that rests on the quartz window. The antenna was driven at $13.56 \mathrm{MHz}$ at 85 mtorr argon pressure and a power of $300 \mathrm{~W}$ measured with a Bird wattmeter between the power supply and matching network.

The photo depicted in Fig. 1 was taken through the quartz window separating the induction antenna from the plasma, with a line of sight almost parallel to the system axis. Because the two are not quite parallel, however, circular objects appear slightly distorted. The antenna loop and an $\mathrm{O}$ ring that seals the quartz plate to an aluminum flange on the top of the vacuum chamber are clearly visible in the photo as dark circles. The

Manuscript received July 17, 1995. This work supported in part by NSF Grant EEC 8721545.

The authors are with the Engineering Research Center for Plasma-Aided Manufacturing and the Electrical and Computer Engineering Department, University of Wisconsin-Madison, Madison, WI 53706 USA.

Publisher Item Identifier S 0093-3813(96)02186-8. quartz plate is held in place by four aluminum clamps. The two ends of the antenna along with connections to external leads are at the top of the photograph. The outer circle in the photograph (outside of which the photo is black) corresponds to a cylindrical aluminum tube extending up from the flange in the plane of the quartz plate and serving as an RF shield. Light that appears to originate between the $\mathrm{O}$ ring and the shield is actually light from the plasma reflecting off the aluminum surfaces. A perforated aluminum sheet parallel to the quartz window, serving as an RF shield between the antenna and the camera is also visible.

\section{RESUlTS AND Discussion}

With the high aspect ratio reactor geometry used in this study, the plasma glow was always restricted to a ring-shaped region with radius slightly smaller than that of the antenna. For the discharge conditions shown in Fig. 1 (300 W RF power, 85 mtorr), 10 striations are apparent, with an approximate spatial period of $7.5 \mathrm{~cm}$. The striations were stationary some of the time, but were also observed to move at random intervals, and completely faded away after $20-30 \mathrm{~min}$ of continuous discharge operation.

Electrostatic trapping of electrons both axially and radially in this discharge is likely to contribute to formation of azimuthal striations. The electron density, $n_{e}$, at and in the vicinity of the discharge axis has been found to be negligible [2], and both $n_{e}$ and the plasma potential peak off-axis in the glowing ring. Thus, electrons are confined in the region of strong azimuthal RF electric field and their motion is channeled electrostatically into the azimuthal direction. This is not the case in lower aspect ratio systems, in which the plasma potential typically has a maximum on the discharge axis. Electron collisions are also likely to play a role in the formation of striations, as striations were not observed at pressures less than 30 mtorr, below which inelastic mean free paths become significant compared to the system length and striation spatial period.

Although striations have not been previously reported for planar RF ICP discharges, they have been observed in other types of discharges in a pressure range that includes the pressure of the case reported here. These include axial striations in the positive columns and azimuthal striations in the anode glow (anode spots) of dc glow discharges [3]. Although both the anode spots observed in dc glow discharges [4] and the ICP striations show structure in the azimuthal direction, the mechanisms are probably quite different. The cause of the ICP striations is more likely to be similar to that of striations observed in positive columns of glow discharges. In the case of the positive column, striations are the result of ionization waves, with feedback between axial modulations 


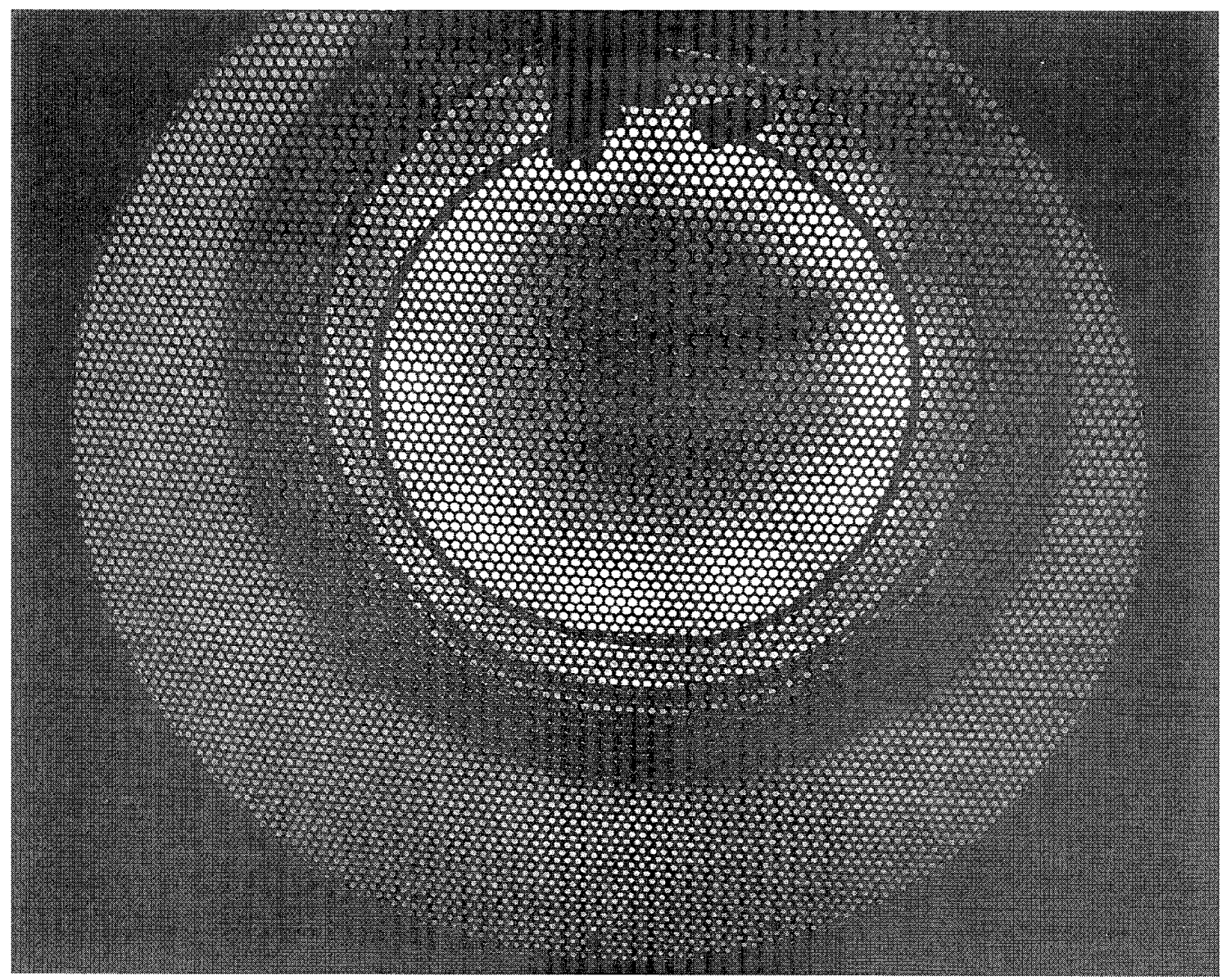

Fig. 1. Azimuthal striations in an RF planar inductively coupled argon plasma. Discharge is viewed photographically through the quartz window through which power is coupled to the plasma, with the single-turn circular antenna clearly visible. Operating conditions are as follows: $300 \mathrm{~W}$ RF power, 85 mtorr pressure, and reactor aspect ratio: radius/height $\approx 7$.

in plasma density, axial dc electric field, electron energy distribution function, and ionization rate. The spatial period of the striation in the positive column corresponds approximately to the distance $\lambda_{\epsilon}$ in the dc electric field an electron must accelerate to acquire kinetic energy equal to the excitation energy. Striations occur when $\lambda_{\epsilon} \gg \lambda^{*}$, the energy relaxation length for electrons in the inelastic energy range, so that once an electron has reached the excitation threshold energy it travels a relatively short distance before giving it up in an inelastic collision.

There are both similarities and differences between the conditions under which striations are observed in the ICP system and the glow discharge positive column. Electric fields in the ICP discharge are directed along the striations, as in the positive column. Field magnitudes in ICP discharges under similar conditions have been found to have peak values of 4-5 V/cm [5], so the requirement that $\lambda_{\epsilon} \gg \lambda^{*}$ is satisfied. However, the temporal oscillation of the ICP induction fields at the RF frequency suggest a significant departure from the positive column. In electric fields of this magnitude, electrons cannot accelerate to the inelastic threshold energy in a single RF cycle. So while the observed ICP striations are likely to arise from ionization waves as in the positive column, there are differences in the mechanism associated with the time oscillation of the RF ICP fields. We therefore conclude that positive column theory, while possibly providing a partial explanation, does not provide a complete description of ICP striations.

\section{ACKNOWLEDGMENT}

The authors gratefully acknowledge helpful discussions with Dr. V. Kolobov.

\section{REFERENCES}

[1] L. J. Mahoney, A. E. Wendt, E. Barrios, C. J. Richards, and J. L. Shohet, "Electron density and energy distributions in a planar inductively coupled discharge," J. Appl. Phys., vol. 76, pp. 2041-2045, 1994.

[2] J. A. Stittsworth and A. E. Wendt, "Chamber geometry and plasma uniformity in a planar inductively coupled radio frequency argon discharge," submitted to Plasma Sources Sci. Technol.

[3] V. I. Kolobov and V. A. Godyak, "Nonlocal electron kinetics in collisional gas discharge plasmas," IEEE Trans. Plasma Sci., vol. 23, no. 4, pp. 503-531, Aug. 1995.

[4] Y. B. Goluborskii, V. I. Kolobov, and S. K. al-Hawat, "Anode region of low-current glow discharges at low and high pressures," Sov. Phys. Tech. Phys., vol. 35, pp. 747-749, 1990.

[5] J. A. Meyer and A. E. Wendt, "Measurements of electromagnetic fields in a planar radio-frequency inductively coupled plasma source," J. Appl. Phys., vol. 78, pp. 90-96, 1996 